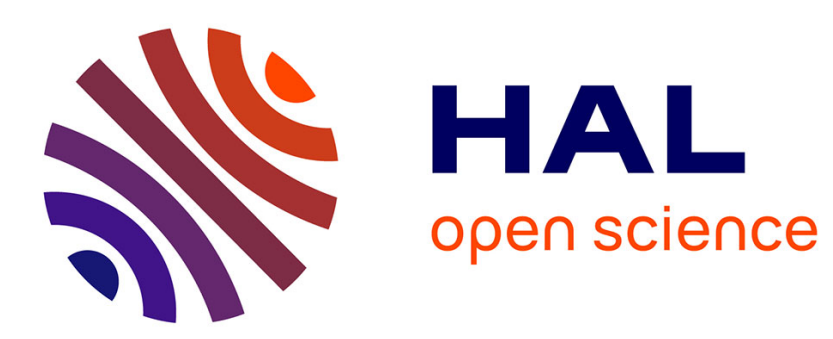

\title{
Empirical Estimation of Tail Dependence Using Copulas. Application to Asian Markets
}

Cyril Caillault, Dominique Guegan

\section{To cite this version:}

Cyril Caillault, Dominique Guegan. Empirical Estimation of Tail Dependence Using Copulas. Application to Asian Markets. Quantitative Finance, 2005, 5, pp.489 - 501. halshs-00180865

\section{HAL Id: halshs-00180865 \\ https://shs.hal.science/halshs-00180865}

Submitted on 22 Oct 2007

HAL is a multi-disciplinary open access archive for the deposit and dissemination of scientific research documents, whether they are published or not. The documents may come from teaching and research institutions in France or abroad, or from public or private research centers.
L'archive ouverte pluridisciplinaire HAL, est destinée au dépôt et à la diffusion de documents scientifiques de niveau recherche, publiés ou non, émanant des établissements d'enseignement et de recherche français ou étrangers, des laboratoires publics ou privés. 


\title{
Empirical Estimation of Tail Dependence Using Copulas. Application to Asian Markets
}

\section{Cyril Caillault $\uparrow$ and Dominique Guégan $\ddagger$}

† Ecole Normale Supérieure, Cachan, Adress: 61, avenue du président Wilson, 94230 Cachan and Dexia Crédit Local, 7 à 11, quai André Citroën BP1002 75901 Paris cedex 15 France

E-mail: cyril.caillault@clf-dexia.com

$\ddagger$ Ecole Normale Supérieure, Cachan, Senior Academic Fellow de l’Institut Europlace de finance (IEF). Adress: 61, avenue du président Wilson, 94230 Cachan, France

E-mail: guegan@ecogest.ens-cachan.fr

\begin{abstract}
This paper introduces non-parametric estimators for upper and lower tail dependence whose confidence intervals are obtained with a bootstrap method. We call these estimators "Naïve estimators" as they represent a discretization of Joe's formulae (1997) linking copulas to tail dependence. We apply the methodology to an empirical data set composed of three composite indexes for the three Tigers (Thailand, Malaysia and Indonesia). The extremes show a dependence structure which is symmetric for the Thai and Malaysian markets and asymmetric for the Thai and Indonesian markets and for the Malaysian and the Indonesian markets. Using these results we estimate the copula (which belongs to the Student or Archimedean copula families) for each pair of markets by two methods. Finally, we provide risk measurements using the best copula associated to each pair of markets.
\end{abstract}

Keywords: Copulas; Tail dependence; Non-parametric Estimation; Semi-parametric estimation; Bootstrap; Risk's measure.

Submitted to: Quantitative Finance

PACS number: RISK MAN 


\section{Introduction}

The existence of co-movements and contagion in detrended macroeconomic series are important issues that have been discussed in the literature. Mainly, it was shown that the cross market correlation increases up to a significant degree during turmoil periods. This means that if a shock affects one market (decreasing for instance), this has a domino effect on the other one (which also decreases). In this article, we are interested in Asian markets and particularly in the three Tigers. The period of study includes the crisis at the end of the Nineties, which represented a period of great turbulence. Rather than investigating this contagion's phenomenon, we will focus on the existence of interaction between these markets. First of all the knowledge of interaction between the markets necessitates to measure their correlation. It is well known that the correlation is only informative in a linear and a Gaussian context. Moreover it can change, see Boyer et al. (1999), because the volatility evolves overtime. To circumvent this problem we will use the tail correlation measure. This measure is very interesting because it provides a global information and takes into account the existence of dependence inside extreme values of data sets. It also permits risk management and proposes a measure of risk for a portfolio.

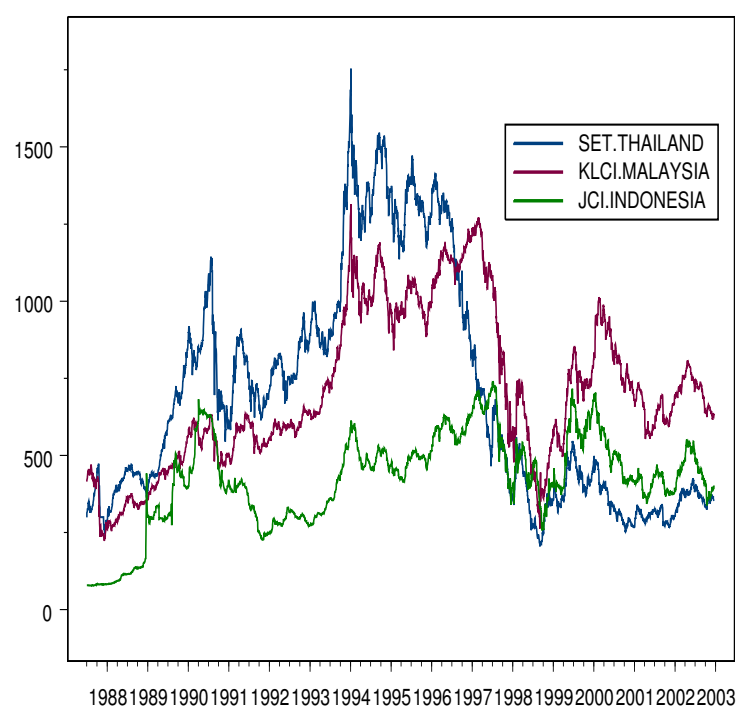

Figure 1. Daily closing level of Asian markets from 02/07/1987 to 17/12/2002. Data were collected at Dexia Crédit Local via Bloomberg.

The understanding of the interaction between different markets requires knowledge of the joint distribution function for two data sets. The latter is difficult to obtain when we are not in an independent setting, but as soon as the variables are dependent we can use the copulas to approach the joint distribution. Let $\left(Z_{1}, \ldots, Z_{n}\right)$ be $n$ markets, we are going to obtain the copulas which characterize them. This approach is not new and a lot of researchers have already investigated this problem, see for instance Frees and Valdez (1998) and Breymann et al. (2003). These articles show the opportunity 
to leave the Gaussian world, as is pointed out in Embrechts et al. (1999, 2002). Our approach will be different: we will use the tail dependence behavior of the markets to determine the best copula for each pair of markets.

We recall that a copula $C$ links multivariate distribution function $F$ to its unidimensional marginal distribution functions $\left(F_{1}, \ldots, F_{n}\right)$ thanks to the following relationship due to Sklar's theorem (1959):

$$
\forall z_{1}, \ldots, z_{n} \in \mathbb{R} \quad F\left(z_{1}, \ldots, z_{n}\right)=C\left(F_{1}\left(z_{1}\right), \ldots, F_{n}\left(z_{n}\right)\right) .
$$

Function $C$ is unique if and only if $F_{1}, \ldots, F_{n}$ are continuous.

In this paper, in order to find the best copula which characterizes the markets that we investigate, we consider two approaches:

(i) We develop a method which uses the empirical copula of Deheuvels (1979), and the notion of tail dependence. We introduce new estimators for the upper and the lower tail dependence, respectively $\hat{\lambda}_{U}$ and $\hat{\lambda}_{L}$. We use these estimates to determine the copula which is best adapted to the data. These tail estimates are called "Naïve" estimators because they represent a discretization of Joe's formula linking copulas to tail dependence (see Joe (1997), page 33 for their precise definition). Other works are related to the estimate of the tail dependence: Malevergne and Sornette (2001, 2002) investigated the null hypothesis that the dependence between financial assets can be modeled by the Gaussian copula. Using the factor models' framework, they established a general expression of the coefficient for the tail dependence between a market and a stock; Coles et al. (1999) proposed an estimation of the tail dependence using a logarithm approximation. The difference with our approach is that it is based on the definition that we use to derive an estimation of $\lambda_{U}$ and $\lambda_{L}$. We also propose some simulations to specify the properties of these estimates and we investigate the influence of the sample size. This approach permits to characterize the estimated copulas with respect to their tail dependence behavior.

(ii) For the different copulas chosen amongst Elliptical and Archimedean families, we estimate their parameters using a maximum likelihood approach, see also Oakes (1994) and Breymann et al. (2003). This approach is based on the Akaike criteria (Akaike, (1974)), to discriminate between the different copulas. This last approach permits us to derive estimates for tail dependence.

These two methods are complementary. The first one uses both tail dependence behavior of the bivariate distribution and a $D_{2}$ diagnostic to discriminate between a set of copulas. The second one is based on the maximum likelihood estimate of the copula's parameter $\psi$. The first approach is based on the local properties of the bivariate distribution and the second one on its global properties.

The remainder of the paper is organized as follows. In Section 2, we define the concept of tail dependence and we introduce the Student and Archimedean copulas. 
Section 3 introduces the "Naïve" estimators for the upper and lower tail dependence parameters. We explain how these parameters work empirically and we derive their values for Gumbel and Clayton copulas through simulations. We apply this method to compute the tail dependence of Tiger markets pairs. In Section 4 we introduce two different methodologies to find the "best" copula which characterizes the market pairs. We compare the methods. In Section 5, these results are used to define a measure of risk for each markets pair. Finally, Section 6 concludes.

\section{Tail Dependence and Copulas}

In this Section we introduce the tail dependence concept. We define Student and Archimedean copulas and provide relationships between Kendall's tau, the correlation's coefficient and the tail dependence parameters for these copula families.

\subsection{Tail dependence concept}

In order to estimate the copula from a bivariate observational data sets, we use in Section 3 the tail dependence concept. It relates the amount of dependence in the upper-rightquadrant tail or in the lower-left-quadrant tail of a bivariate distribution. The upper and lower tail dependence parameters of the random vector $\left(Z_{1}, Z_{2}\right)$ with copula $C$, can be defined in the following way, see Joe (1997) for instance:

Definition 2.1 If a bivariate copula $C$ is such that

$$
\lim _{u \uparrow 1} \bar{C}(u, u) /(1-u)=\lambda_{U}
$$

exists, with $\bar{C}(u, u)=1-2 u+C(u, u)$, then the copula $C$ has an upper tail dependence if $\lambda_{U} \in(0,1]$ and no upper tail dependence if $\lambda_{U}=0$. Moreover if a bivariate copula $C$ is such that

$$
\lim _{u \downarrow 0} C(u, u) / u=\lambda_{L}
$$

exists, we will say that the copula $C$ has lower tail dependence if $\lambda_{L} \in(0,1]$ and no lower tail dependence if $\lambda_{L}=0$.

This Definition will be used in Section 3 to derive estimators for the tail dependence parameters.

\subsection{Student Copula}

In this article, the data sets will led us to use the Student copula. This copula belongs to the elliptical family and is completely described by: the linear correlation coefficient $\rho$ and the degrees of freedom $\nu$. For this symmetrical copula we have a relationship between $\rho$ and Kendall's tau, see Lindskog et al. (2001):

$$
\tau\left(Z_{1}, Z_{2}\right)=\frac{2}{\pi} \arcsin \left(\rho\left(Z_{1}, Z_{2}\right)\right) .
$$


Then, the lower and upper tail dependence coefficients for the Student copula are given by the following equation:

$$
\lambda_{L}=\lambda_{U}=2 \bar{t}_{\nu+1}\left(\sqrt{\frac{(\nu+1)(1-\rho)}{1+\rho}}\right),
$$

where $\bar{t}_{\nu+1}(x)=1-t_{\nu+1}(x)$ and $t_{\nu+1}$ is the Student distribution function with $\nu+1$ degrees of freedom. Thus, $\lambda_{U}$ is an increasing function of $\rho$. We can also observe that when $\nu$ tends to infinity, $\lambda_{U}$ tends to 0 . For more details on the elliptical copulas, we refer to Cambanis et al. (1981) and Fang et al. (2002).

\subsection{Archimedean Copulas}

Archimedean copulas are defined through a generator function $\phi_{\theta}$ such that:

$$
C_{\phi_{\theta}}(u, v)=\phi_{\theta}^{-1}\left(\phi_{\theta}(u)+\phi_{\theta}(v)\right),
$$

where $\phi_{\theta}:[0,1] \rightarrow\left[0, \infty\left[\right.\right.$ is a decreasing convex function such that $\phi_{\theta}(1)=0$, with $\phi_{\theta}(0)=\lim _{t \downarrow 0} \phi_{\theta}(t)$ and $\phi_{\theta}^{-1}(t)=0$ for $s \geq \phi_{\theta}(0)$, by convention. When $\lim _{t \downarrow 0} \phi_{\theta}(t)=\infty$ we say that $\phi_{\theta}$ is a strict generator and that $C_{\phi_{\theta}}$ is a strict copula.

In Table 1 we specify the range of the parameter $\theta$, the tail dependence parameters and the Kendall's tau of the following copulas: Clayton, Frank, Gumbel and Joe. These results constitute a little part of those given by Genest and MacKay (1986a,b), Ledford et al. (1996, 1998), Joe (1997), Heffernan (2000) and Caillault and Guégan (2003). Note that $\tau, \lambda_{U}$ and $\lambda_{L}$ are expressed in function of $\theta$. We can also derive $\lambda_{U}$ and $\lambda_{L}$ of a survival copula f from its associated copula using the Definition 2.1. Thus, $\lambda_{U}^{C_{S}}=\lambda_{L}^{C}$ and $\lambda_{L}^{C_{S}}=\lambda_{U}^{C}$. It means that if a copula has an upper tail dependence then the associated survival copula has a lower tail dependence and conversely. Moreover, a survival copula and its associated copula have the same Kendall's tau, see Georges et al. (2001) for details.

Table 1. Properties of Archimedean Copulas. (a) only true for $\theta>0$.

$D_{1}(\theta)=\frac{1}{\theta} \int_{0}^{\theta} \frac{t}{e^{t}-1} d t$. The symbol $\checkmark$ means that a numerical computation is required to obtain $\tau$.

\begin{tabular}{rcccc}
\hline Family & Range of $\theta$ & $\lambda_{U}$ & $\lambda_{L}$ & $\tau$ \\
\hline Clayton & {$[-1,+\infty[\backslash\{0\}$} & 0 & $2^{-\frac{1}{\theta}}(a)$ & $\frac{\theta}{\theta+2}$ \\
Frank & ]$-\infty,+\infty[\backslash\{0\}$ & 0 & 0 & $1+4\left(D_{1}(\theta)-1\right) / \theta$ \\
Gumbel & {$[1,+\infty[$} & $2-2^{\frac{1}{\theta}}$ & 0 & $1-\frac{1}{\theta}$ \\
Joe & {$[1,+\infty[$} & $2-2^{\frac{1}{\theta}}$ & 0 & $\checkmark$ \\
\hline
\end{tabular}

$\ddagger$ The survival copula of $C$ is given by: $C_{S}(u, v)=u+v-1+C(1-u, 1-v)$. 
The Archimedean copulas introduce above cannot explain all the tail behavior observed on financial markets. Indeed, looking at Table 1, we observe that if a copula has an upper tail dependence then it does not have a lower tail dependence and conversely. In order to obtain copulas which have an upper and a lower tail dependence without being symmetrical, we construct new copulas as a convex linear combination of two copulas. Hence, for $\omega \in[0,1]$ and two Archimedean copulas $C_{\theta_{1}}$ and $C_{\theta_{2}}$ we obtain a copula $C$ defined as:

$$
C(u, v)=\omega C_{\theta_{1}}(u, v)+(1-\omega) C_{\theta_{2}}(u, v) .
$$

The properties of these copulas can be derived from those of $C_{\theta_{1}}$ and $C_{\theta_{2}}$. Suppose that $C_{\theta_{1}}$ and $C_{\theta_{2}}$ have respectively an upper and a lower tail dependence. Then,

$$
\lambda_{U}^{C}=\omega \lambda_{U}^{C_{\theta_{1}}},
$$

and

$$
\lambda_{L}^{C}=(1-\omega) \lambda_{L}^{C_{\theta_{2}}}
$$

The Kendall's tau of the copula $C$ defined in (2.4) is equals to§:

$$
\tau_{C}=\tau_{C}=\omega^{2} \tau_{C_{\theta_{1}}}+(1-\omega)^{2} \tau_{C_{\theta_{2}}}+2 \omega(1-\omega)\left(4 \int_{I^{2}} C_{\theta_{1}}(u, v) d C_{\theta_{2}}(u, v)-1\right) .
$$

The relationship (2.7) can also be expressed in function of the parameters $\lambda_{U}^{C}, \lambda_{L}^{C}$ and $\omega$. Assume that $\theta_{1}$ and $\theta_{2}$ are respectively the parameters of the Gumbel and Clayton copulas, then if $C$ represents their convex linear combination as in (2.4), we obtain for $\omega \neq 0,1$ that:

$$
\tau_{C}=\omega\left(1-1 / \theta_{1}\right)+(1-\omega)\left(\theta_{2} /\left(\theta_{2}+2\right)\right)+2 \omega(1-\omega)\left(4 \int_{I^{2}} C_{\theta_{1}}(u, v) d C_{\theta_{2}}(u, v)-1\right),(2 .
$$

where

$$
\theta_{1}=\frac{\log 2}{\log \left(2-\lambda_{U}^{C} / \omega\right)}
$$

and

$$
\theta_{2}=-\frac{\log 2}{\log \left(\lambda_{L}^{C} /(1-\omega)\right)}
$$

Then, for given $\tau_{C}, \lambda_{U}^{C}$ and $\lambda_{L}^{C}, \omega$ is deduced from (2.8), and $\theta_{1}$ and $\theta_{2}$ are obtained from equations (2.9) and (2.10). We can derived similar formulae for the convex linear combination of: Gumbel + Survival Gumbel, Survival Clayton + Survival Gumbel and Survival Clayton + Clayton. By convention, the first copula for all the convex linear combinations has an upper tail dependence and the second one has a lower tail dependence.

$\S$ We make a point of thanking Jean-David Fermanian whose remarks made it possible to obtain this result. $\int_{I 2} C_{\theta_{1}}(u, v) d C_{\text {theta }_{2}}(u, v)$ is obtained numerically by using the Fortran library ADAPT developed by A. Genz (See Berntsen et al. $(1991 \mathrm{a}, \mathrm{b})$ ). 


\section{Estimation of tail dependence: Application to Asian Markets}

In this section, we introduce the "Naïve" estimators for the upper and lower tail dependence parameters derived from Definition 2.1 and based on the Deheuvels' copula (1979). We specify the properties of these estimators through simulations. In fine, we apply our results to three Asian markets.

\section{1. "Naïve" Estimation for the tail dependence parameters}

In order to adjust a copula from an observational data sets, we choose to model the extremes of each markets pair, via the parameters $\lambda_{U}$ and $\lambda_{L}$. In this way we propose the following estimators for $\lambda_{U}$ and $\lambda_{L}$ called "Naïve" estimators. Their representation are:

$$
\hat{\lambda}_{U}=\lim _{i \rightarrow N} \frac{1-\frac{2 i}{N}+\hat{C}\left(\frac{i}{N}, \frac{i}{N}\right)}{1-\frac{i}{N}}
$$

and

$$
\hat{\lambda}_{L}=\lim _{i \rightarrow 0} \frac{\hat{C}\left(\frac{i}{N}, \frac{i}{N}\right)}{\frac{i}{N}},
$$

where $N$ is the length of the data sets. The empirical copula $\hat{C}$ introduced by Deheuvels (1979) is defined as follows:

Definition 3.1 Let $\mathbf{z}=\left\{\left(z_{1_{k}}, z_{2_{k}}\right)\right\}_{k=1}^{N}$ denotes a sample of size $N$ from a continuous bivariate distribution. The empirical copula is the function $\hat{C}$ defined by:

$$
\hat{C}\left(\frac{i}{N}, \frac{j}{N}\right)=\frac{\#\left(\left(z_{1}, z_{2}\right), z_{1} \leq z_{1}^{(i)} \text { and } z_{2} \leq z_{2}^{(j)}\right)}{N},
$$

where \# is used for cardinal and $z_{1}^{(i)}, z_{2}^{(j)}$ for $1 \leq i, j \leq N$ represent the order statistics obtained from the sample.

This empirical distribution $\hat{C}$ is uniformly almost surely consistent, see Deheuvels (1979). This property can be extended to $\hat{\lambda}_{U}$ and $\hat{\lambda}_{L}$. Limits (3.1) and (3.2) cannot be obtained in an empirical setting. Thus, we introduce the following procedure to obtain estimates of $\lambda_{U}$ and $\lambda_{L}$.

We define the two next statistics for $i \in\{1, \ldots, N-1\}$ :

$$
\hat{\lambda}_{U}\left(\frac{i}{N}\right)=\frac{1-\frac{2 i}{N}+\hat{C}\left(\frac{i}{N}, \frac{i}{N}\right)}{1-\frac{i}{N}}
$$

and

$$
\hat{\lambda}_{L}\left(\frac{i}{N}\right)=\frac{\hat{C}\left(\frac{i}{N}, \frac{i}{N}\right)}{\frac{i}{N}},
$$

with $\hat{C}$ given in (3.3). These statistics represent the trajectory of $\lambda_{U}$ and $\lambda_{L}$ on $[0,1]$ with respect to $i / N$. Now, to obtain the expected values of $\hat{\lambda}_{U}$ and $\hat{\lambda}_{L}$ defined in (3.1) 
and (3.2), we are going to choose the position $i$ graphically. We propose to select $i$ using a specific property of $\lambda_{U}$ and $\lambda_{L}$. We know that on $[0,1], \lambda_{U}$ and $\lambda_{L}$ are a decreasing and an increasing function of $u$, thus the last values of $i=i_{0}$ for which these properties are observed, will be chosen. Then, we define:

$$
\hat{\lambda}_{U}=\hat{\lambda}_{U}\left(\frac{i_{0}}{N}\right)
$$

and

$$
\hat{\lambda}_{L}=\hat{\lambda}_{L}\left(\frac{i_{0}}{N}\right)
$$

Now, if $\hat{\lambda}_{U}($.$) takes identical values for several consecutive values i$, then we record these $k$ values which are include between $N-p-k$ and $N-p$ and we choose $i_{0}$ as the median of the interval (the same method is applied with $\left.\hat{\lambda}_{L}().\right)$. The principal difficulty of the previous mechanical procedure lies in the interpretation of the variation's change or the stability of the curves $\hat{\lambda}_{U}($.$) and \hat{\lambda}_{L}($.$) . Suppose that we select i$ too large, then the $\hat{\lambda}_{U}$ confidence interval could explode. Now, if $i$ is selected too small, then we introduce a bias in the estimates. Only the practice and the following step permit to obtain a useful approach to choice $i_{0}$.

This step consists to obtain the average curves $\lambda_{U}^{*}($.$) and \lambda_{L}^{*}($.$) which are smoother than$ $\hat{\lambda}_{U}($.$) and \hat{\lambda}_{L}($.$) . They are obtained by a bootstrap method. After s$ bootstrap resampling $\mathbf{z}^{*}$, we obtain $s$ trajectories from which we derive the average curves. Then, we use the method based on percentiles of the bootstrap distribution introduced in Efron and Tibshirani (1993) to obtain confidence intervals of $\hat{\lambda}_{U}\left(\frac{i}{N}\right)$ and $\hat{\lambda}_{L}\left(\frac{i}{N}\right)$. The bounds of these confidence intervals at level $1-2 \alpha$ are the quantile of the bootstrap distributions. We denote them by $\hat{\lambda}_{U}^{(l o w)}\left(\frac{i}{N}\right)=Q_{\alpha}$ and $\hat{\lambda}_{U}^{(u p)}\left(\frac{i}{N}\right)=Q_{1-\alpha}$. This step permits to choose more precisely the value $i_{0}$ for which we observe a behavior's change.

Now, we provide simulations. We simulated $N=100,5000$ samples from the Gumbel copula with standard Gaussian margins, for several values of the parameter $\lambda_{U}$ and $s=100$. The values obtained for $\lambda_{U}$ using the previous method, are given in Table 2 . This table provides the values of $i_{0}$, and the column called "Observed" corresponds to the values of upper tail dependence estimate obtained without bootstrap. The other columns correspond to the mean, the standard error (s.e), the bias and the quantiles of the empirical bootstrap distribution function of $\hat{\lambda}_{U}\left(\frac{i_{0}}{N}\right)$ with $\alpha=5 \%$. We observe that the bigger $N$ is, the more efficient is the estimate for the upper tail dependence parameter. Indeed, we note that the standard error and the bias decrease with the increase of the sample length. On the other hand, the convergence towards the true value seems difficult to obtain when $\lambda_{U}$ is lower than 0.2 , whatever the sample size $N$. This Table also gives the lower tail dependence parameter estimates for the Clayton copula. The plan of simulations is the same as before. Again, we observe that the estimates improve as soon as $N$ is bigger. 
Table 2. Empirical estimation of the upper tail dependence parameter of the Gumbel copula and of the lower tail dependence parameter of the Clayton copula using the "Naïve" estimators.

\begin{tabular}{|c|c|c|c|c|c|c|c|c|}
\hline & $\lambda_{U}$ & $i_{0}$ & Observed & Mean & s.e & Bias & $Q_{5 \%}$ & $Q_{95 \%}$ \\
\hline \multirow{10}{*}{$\begin{array}{l}\text { Gumbel Copula } \\
N=100 s=100\end{array}$} & 0.1 & 97 & 0.00 & 0.16 & 0.25 & -0.16 & 0.00 & 0.67 \\
\hline & 0.2 & 94 & 0.17 & 0.23 & 0.19 & -0.06 & 0.00 & 0.50 \\
\hline & 0.3 & 86 & 0.29 & 0.36 & 0.10 & -0.07 & 0.21 & 0.57 \\
\hline & 0.4 & 90 & 0.42 & 0.44 & 0.13 & -0.02 & 0.25 & 0.67 \\
\hline & 0.5 & 80 & 0.48 & 0.54 & 0.09 & -0.06 & 0.38 & 0.67 \\
\hline & 0.6 & 82 & 0.65 & 0.62 & 0.10 & 0.03 & 0.45 & 0.80 \\
\hline & 0.7 & 79 & 0.73 & 0.72 & 0.10 & 0.00 & 0.55 & 0.86 \\
\hline & 0.8 & 77 & 0.76 & 0.77 & 0.07 & -0.01 & 0.68 & 0.88 \\
\hline & 0.9 & 89 & 0.85 & 0.87 & 0.08 & -0.02 & 0.69 & 1.00 \\
\hline & 0.95 & 76 & 0.96 & 0.95 & 0.04 & 0.01 & 0.88 & 1.00 \\
\hline \multirow{11}{*}{$\begin{array}{l}\text { Gumbel Copula } \\
N=5000 \quad s=100\end{array}$} & 0.1 & 4955 & 0.07 & 0.10 & 0.05 & -0.03 & 0.05 & 0.19 \\
\hline & 0.2 & 4925 & 0.19 & 0.20 & 0.05 & -0.01 & 0.13 & 0.29 \\
\hline & 0.3 & 4808 & 0.29 & 0.28 & 0.03 & 0.01 & 0.23 & 0.33 \\
\hline & 0.4 & 4819 & 0.40 & 0.40 & 0.03 & 0.00 & 0.35 & 0.46 \\
\hline & 0.5 & 4865 & 0.51 & 0.51 & 0.04 & 0.00 & 0.44 & 0.57 \\
\hline & 0.6 & 4724 & 0.58 & 0.58 & 0.02 & 0.00 & 0.57 & 0.63 \\
\hline & 0.7 & 4638 & 0.71 & 0.72 & 0.02 & -0.01 & 0.68 & 0.75 \\
\hline & 0.8 & 4678 & 0.81 & 0.80 & 0.02 & 0.00 & 0.77 & 0.84 \\
\hline & 0.9 & 4689 & 0.90 & 0.90 & 0.01 & 0.00 & 0.87 & 0.92 \\
\hline & 0.95 & 4756 & 0.95 & 0.95 & 0.01 & 0.00 & 0.93 & 0.97 \\
\hline & $\lambda_{L}$ & $i_{0}$ & Observed & Mean & s.e & Bias & $Q_{5 \%}$ & $Q_{95 \%}$ \\
\hline \multirow{10}{*}{$\begin{array}{l}\text { Clayton Copula } \\
N=100 \quad s=100\end{array}$} & 0.1 & 7 & 0.14 & 0.18 & 0.17 & -0.04 & 0.00 & 0.43 \\
\hline & 0.2 & 9 & 0.22 & 0.25 & 0.13 & -0.03 & 0.11 & 0.44 \\
\hline & 0.3 & 17 & 0.18 & 0.24 & 0.09 & -0.06 & 0.12 & 0.41 \\
\hline & 0.4 & 7 & 0.43 & 0.36 & 0.18 & 0.07 & 0.00 & 0.71 \\
\hline & 0.5 & 21 & 0.52 & 0.55 & 0.09 & -0.02 & 0.38 & 0.71 \\
\hline & 0.6 & 16 & 0.56 & 0.57 & 0.11 & -0.01 & 0.38 & 0.75 \\
\hline & 0.7 & 27 & 0.68 & 0.68 & 0.09 & 0.00 & 0.54 & 0.82 \\
\hline & 0.8 & 29 & 0.83 & 0.84 & 0.07 & -0.01 & 0.72 & 0.93 \\
\hline & 0.9 & 20 & 0.85 & 0.87 & 0.06 & -0.02 & 0.75 & 1.00 \\
\hline & 0.95 & 12 & 0.92 & 0.88 & 0.11 & 0.03 & 0.67 & 1.00 \\
\hline \multirow{10}{*}{$\begin{array}{l}\text { Clayton Copula } \\
N=5000 \quad s=100\end{array}$} & 0.1 & 61 & 0.16 & 0.16 & 0.04 & 0.00 & 0.10 & 0.23 \\
\hline & 0.2 & 107 & 0.24 & 0.23 & 0.04 & 0.01 & 0.16 & 0.29 \\
\hline & 0.3 & 136 & 0.32 & 0.33 & 0.04 & 0.00 & 0.27 & 0.39 \\
\hline & 0.4 & 230 & 0.40 & 0.41 & 0.03 & 0.00 & 0.37 & 0.45 \\
\hline & 0.5 & 293 & 0.49 & 0.49 & 0.03 & 0.00 & 0.45 & 0.54 \\
\hline & 0.6 & 182 & 0.61 & 0.60 & 0.03 & 0.00 & 0.56 & 0.66 \\
\hline & 0.7 & 162 & 0.70 & 0.69 & 0.03 & 0.01 & 0.64 & 0.74 \\
\hline & 0.8 & 181 & 0.80 & 0.80 & 0.02 & -0.01 & 0.77 & 0.85 \\
\hline & 0.9 & 174 & 0.92 & 0.92 & 0.02 & 0.01 & 0.89 & 0.95 \\
\hline & 0.95 & 234 & 0.94 & 0.94 & 0.00 & 0.00 & 0.93 & 0.95 \\
\hline
\end{tabular}

\subsection{Empirical Study: Application to Asian Markets}

We propose an empirical application which aims at modeling the joint distribution of $n=2$ Asian markets through Student and Archimedean copulas. Our approach consists 
to estimate directly the tail dependence parameters $\lambda_{U}$ and $\lambda_{L}$ for the markets pairs using the "Naïve" estimators introduced previously. The estimates obtained for $\lambda_{U}$ and $\lambda_{L}$ will be used to obtain the parameters of a set of selected copulas.

We consider the three composite indexes of financial Asian places represented in the Introduction. They consist of the daily closing level of the Thai SET index, the Malaysian KLCI index, and the Indonesian JCI index from the 2nd of July 1987 to the 17th of December 2002 with a total of $N=4034$ points. The data sets have been collected from Bloomberg and was expressed in Japanese currency. Then, we considered the log-returns to achieve stationarity. These three series are denoted $\left(Z_{1}\right)_{t}$ for the Thai SET log-returns, $\left(Z_{2}\right)_{t}$ for the Malaysian KLCI log-returns and $\left(Z_{3}\right)_{t}$ for the Indonesian JCI log-returns. Figure 2 provides the trajectories and the histograms for the three log-returns indexes on the full period from 1987 to 2002.

In Table 3, we summarize statistics from these three series. They have a distribution law which is nearly symmetrical and all of them exhibit excess kurtosis relatively to the Gaussian distribution law. Thus, the three series are not Gaussian and we choose to fit them by Laplace distribution laws. It was the best ajustement we obtained compared with other distributions such as a variance gamma or the normal inverse Gaussian ones. These choices are confirmed using the Kolmogorov-Smirnov test with the $95 \%$ level. The values of the parameters of the Laplace distributions for $\left(Z_{1}\right)_{t},\left(Z_{2}\right)_{t}$ and $\left(Z_{3}\right)_{t}$ are respectively: $a=-1.34 \times 10^{-4}$ and $b=1.47 \times 10^{-2}, a=-4.33 \times 10^{-5}$ and $b=1.40 \times 10^{-2}$ and $a=-1.00 \times 10^{-4}$ and $b=1.18 \times 10^{-2}$. In Figure 3, we give the scatter plots corresponding to the three markets pairs on which we want to adjust a copula.

Table 3. Descriptive statistics for $\left(Z_{1}\right)_{t},\left(Z_{2}\right)_{t}$ and $\left(Z_{3}\right)_{t}$.

\begin{tabular}{lrrr}
\hline & $\left(Z_{1}\right)_{t}$ & $\left(Z_{2}\right)_{t}$ & $\left(Z_{3}\right)_{t}$ \\
\hline Mean & $-1.34 \times 10^{-4}$ & $-4.33 \times 10^{-5}$ & $-1.00 \times 10^{-4}$ \\
Standard deviation & 0.020 & 0.019 & 0.026 \\
Kurstosis & 5.897 & 26.928 & 40.391 \\
Skweness & 0.014 & 0.023 & 0.838 \\
Length of the sample & 4033 & 4033 & 4033 \\
\hline
\end{tabular}

From expressions (3.4) and (3.5) we obtained the estimates of the tail dependence parameters $\lambda_{U}$ and $\lambda_{L}$, for $i=\{1, \cdots, N-1\}$ and we represent their trajectory in Figure 4. The three pairs seem to have an upper and a lower tail dependence. In order to find the stable value for $\hat{\lambda}_{U}$ and $\hat{\lambda}_{L}$, we focus on the tail and we try to find a region where these values $\hat{\lambda}_{U}\left(\frac{i}{N}\right)$ and $\hat{\lambda}_{L}\left(\frac{i}{N}\right)$ are stable. Then, we determine the values of the parameter $i_{0}$ following the method developed in 3.1. Formulae (3.6) and (3.7) permit to obtain an estimate for $\lambda_{U}$ and $\lambda_{L}$ for each pair of markets. These values are given in Table 4 . The confidence intervals are computed at the $90 \%$ level.

The results in Table 4, suggest that: 

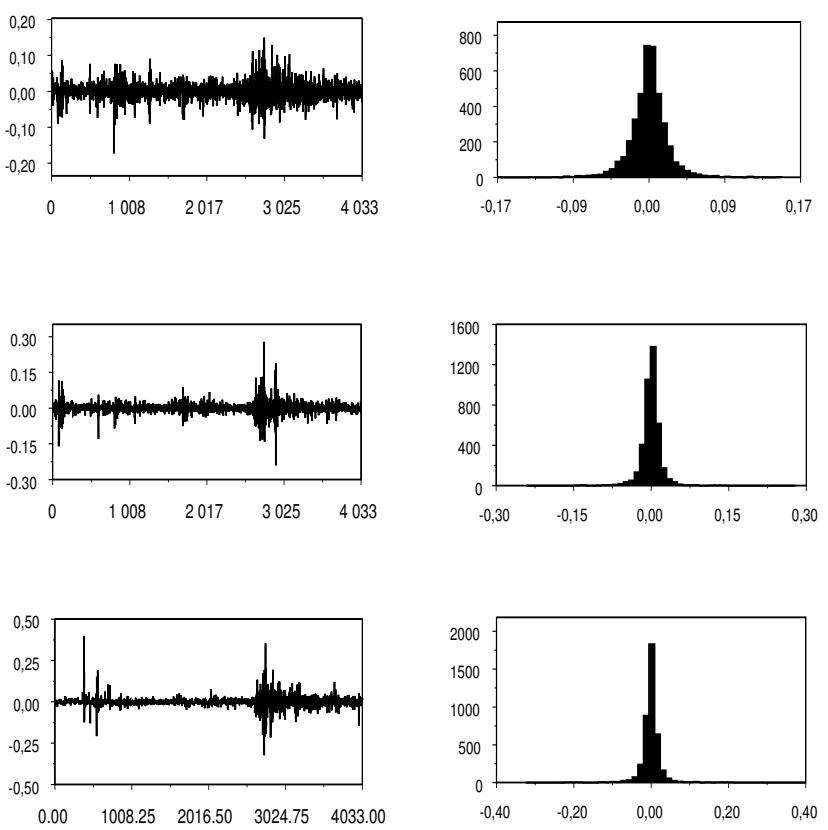

Figure 2. Trajectories and histograms of $\left(Z_{1}\right)_{t},\left(Z_{2}\right)_{t}$ and $\left(Z_{3}\right)_{t}$. The three series are expressed in Japanese currency.

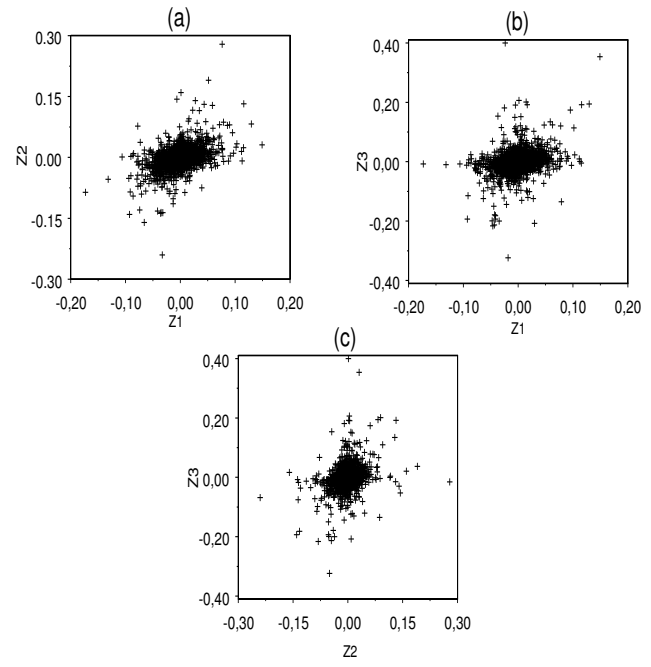

Figure 3. Scatter plot for the pairs $\left(\left(Z_{1}\right)_{t},\left(Z_{2}\right)_{t}\right),\left(\left(Z_{1}\right)_{t},\left(Z_{3}\right)_{t}\right)$ and $\left(\left(Z_{2}\right)_{t},\left(Z_{3}\right)_{t}\right)$.

(i) The pair $\left(\left(Z_{1}\right)_{t},\left(Z_{2}\right)_{t}\right)$ has both an upper and a lower tail dependence. We observe also a symmetrical behavior in the tails. This means that we can adjust a Student copula on this pair of markets .

(ii) The pairs $\left(\left(Z_{1}\right)_{t},\left(Z_{3}\right)_{t}\right)$ and $\left(\left(Z_{2}\right)_{t},\left(Z_{3}\right)_{t}\right)$ seem to be asymmetric in the tails. Thus, in that case, the convex linear combinations of Archimedean copulas are appropriate. 

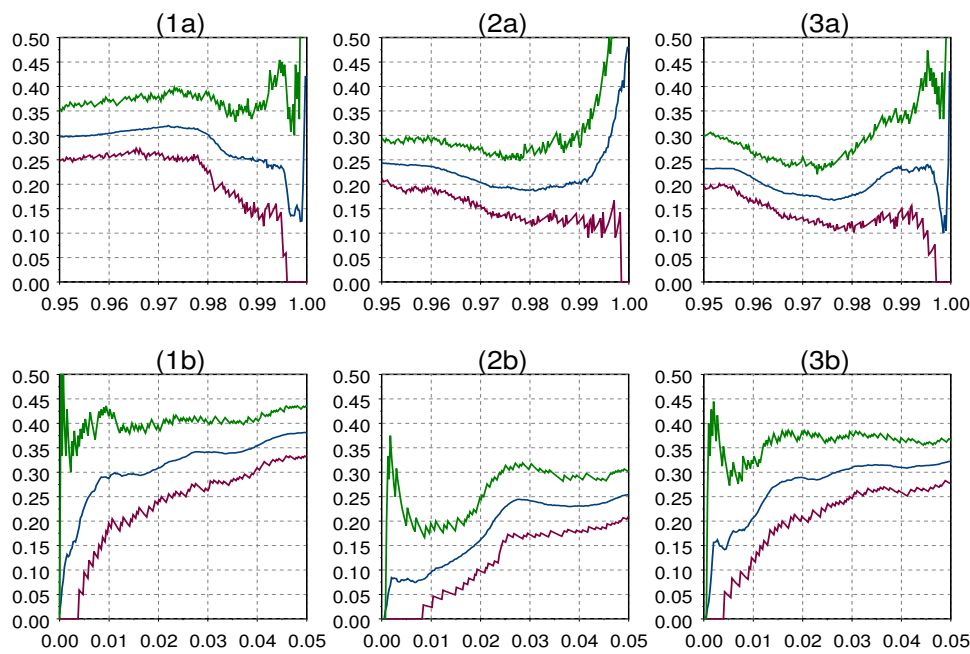

Figure 4. Tail dependence plot. Figures (1a), (2a) and (3a) represent the upper tail dependence of the pairs $\left(\left(Z_{1}\right)_{t},\left(Z_{2}\right)_{t}\right),\left(\left(Z_{1}\right)_{t},\left(Z_{3}\right)_{t}\right)$ and $\left(\left(Z_{2}\right)_{t},\left(Z_{3}\right)_{t}\right)$. Figures $(1 \mathrm{~b})$, $(2 \mathrm{~b})$ and $(3 \mathrm{~b})$ provide the lower tail dependence of each bivariate series.

Table 4. $\hat{\lambda}_{L}$ and $\hat{\lambda}_{U}$ with confidence intervals at the $90 \%$ level for the pairs $\left(\left(Z_{1}\right)_{t},\left(Z_{2}\right)_{t}\right),\left(\left(Z_{1}\right)_{t},\left(Z_{3}\right)_{t}\right)$ and $\left(\left(Z_{2}\right)_{t},\left(Z_{3}\right)_{t}\right)$.

\begin{tabular}{ccccc}
\hline & $i_{0}$ & $i_{0} / N$ & $\hat{\lambda}_{L}$ & Confidence Interval \\
\hline$\left(\left(Z_{1}\right)_{t},\left(Z_{2}\right)_{t}\right)$ & 53 & 0.013 & 0.305 & {$[0.2270 .385]$} \\
$\left(\left(Z_{1}\right)_{t},\left(Z_{3}\right)_{t}\right)$ & 157 & 0.039 & 0.231 & {$[0.1790 .290]$} \\
$\left(\left(Z_{2}\right)_{t},\left(Z_{3}\right)_{t}\right)$ & 89 & 0.022 & 0.284 & {$[0.2130 .370]$} \\
\hline & $i_{0}$ & $i_{0} / N$ & $\hat{\lambda}_{U}$ & Confidence Interval \\
\hline$\left(\left(Z_{1}\right)_{t},\left(Z_{2}\right)_{t}\right)$ & 3835 & 0.951 & 0.302 & {$[0.2630 .342]$} \\
$\left(\left(Z_{1}\right)_{t},\left(Z_{3}\right)_{t}\right)$ & 3954 & 0.980 & 0.187 & {$[0.1190 .273]$} \\
$\left(\left(Z_{2}\right)_{t},\left(Z_{3}\right)_{t}\right)$ & 3939 & 0.977 & 0.167 & {$[0.1050 .244]$} \\
\hline
\end{tabular}

\section{How to discriminate between selected copulas?}

The question now is, how to choose the best copula? We consider two different approaches. One is based on the knowledge of the tail behavior of each pair of markets. In that case, we use the results of Section 3.2. The second one considers a panel of copulas (without any a priori) and we select the best one using the AIC criteria (Akaike (1974)).

\subsection{The $D_{2}$ diagnostic}

To discriminate among the different copulas in according to their tail behavior, we are going to use a $L^{2}$ distance. This one allows us, to measure how far the empirical copula of Deheuvels $\hat{C}$, introduced in (3.3), and the copula $C_{\hat{\psi}}$ estimated from $\hat{\lambda}_{U}$ and $\hat{\lambda}_{L}$ are. $\psi$ is a vector of parameters such as: $\psi=\theta$ for the Archimedean copulas, $\psi=\left(\theta_{1}, \theta_{2}, \omega\right)$ for 
the convex linear combinations of Archimedean copulas and $\psi=(\rho, \nu)$ for the Student copula. Now, we define the $L^{2}$ distance as follows:

$$
D_{2}=\sum_{m=0}^{N} \sum_{n=0}^{N}\left|C_{\hat{\psi}}\left(\hat{F}_{i N}\left(z_{i m}\right), \hat{F}_{j N}\left(z_{j n}\right)\right)-\hat{C}(m / N, n / N)\right|^{2},
$$

where $\hat{F}_{i N}(i=1,2,3)$ is the empirical distribution function:

$$
\hat{F}_{i N}\left(z_{i p}\right)=\frac{1}{N+1} \sum_{k=1}^{N} 1_{\left\{z_{i k}<z_{i p}\right\}},
$$

The copula $C_{\hat{\psi}}$, for which we obtain the minimum distance $D_{2}$, will be chosen as the best approximation, in that sense. This distance is computed using the entire information set with the same weight on each observation: this derived from the use of the Deheuvels copula. It would be interesting to consider other distances which privilege the influence of the points in the tail as an offset to the weight of the center observations of the distribution. But, in that latter case, other estimates for the different distributions which appear in (4.1), are necessary. As a referee points out, we think that it could be interesting to look at this approach with the expectation of making the results proposed here more relevant. Nevertheless, we do not explore this problem here because new estimators for the copulas need to be introduced.

The copulas we considered depend on the tail behavior of the markets pairs. Thus, in according with the results in Section 3.2, we use the Student and also the four convex linear combinations of Archimedean Copulas introduced in Section 2.

To estimate the parameters $\rho$ and $\nu$ of the Student's copula we invert the expression (2.1), $\hat{\rho}\left(Z_{i_{t}}, Z_{j_{t}}\right)=\sin \left(\frac{\pi}{2} \hat{\tau}\left(Z_{i_{t}}, Z_{j_{t}}\right)\right)$ and we obtain $\hat{\nu}$ by solving (2.2) for $\nu$. The empirical estimate $\hat{\tau}$ of $\tau$ is computed with $\hat{\tau}=2 \frac{c-d}{N(N-1)}$, where $c$ is the number of concordant points and $d$ the number of discordant points. The estimate of $\theta_{1}, \theta_{2}$ and $\omega$ are derived from $\hat{\tau}, \hat{\lambda}_{L}$ and $\hat{\lambda}_{U}$, see Section 2.3. Table 5 gives Kendall's tau for each pair of markets, with a confidence interval at a $90 \%$ level. These confidence intervals are based on bootstrap percentiles (see the previous section for details). Table 6 gives the parameters estimates and the $D_{2}$ criteria. For the pair $\left(\left(Z_{1}\right)_{t},\left(Z_{2}\right)_{t}\right)$, we retain the Student copula and for the pairs $\left(\left(Z_{1}\right)_{t},\left(Z_{3}\right)_{t}\right)$ and $\left(\left(Z_{2}\right)_{t},\left(Z_{3}\right)_{t}\right)$ the convex linear combination of a survival Clayton with a Clayton copula.

Table 5. Kendall's tau between Asian markets and the associated confidence intervals

\begin{tabular}{|c|c|c|}
\hline & $\hat{\tau}$ & Confidence Interval \\
\hline$\left(\left(Z_{1}\right)_{t},\left(Z_{2}\right)_{t}\right)$ & 0.292 & {$\left[\begin{array}{ll}0.268 & 0.309\end{array}\right]$} \\
\hline$\left(\left(Z_{1}\right)_{t},\left(Z_{3}\right)_{t}\right)$ & 0.245 & {$\left[\begin{array}{ll}0.225 & 0.262\end{array}\right]$} \\
\hline$\left(\left(Z_{2}\right)_{t},\left(Z_{3}\right)_{t}\right)$ & 0.251 & {$\left[\begin{array}{ll}0.229 & 0.266\end{array}\right]$} \\
\hline
\end{tabular}
at the $90 \%$ level. 
Table 6. Copulas' parameters estimates and $D_{2}$ criteria for each markets pair. For the convex linear combinations of Archimedean copulas the three parameters are $\theta_{1}$, $\theta_{2}, \omega$. For the Student copula the first parameter is the Pearson's correlation $\rho$ and the second one corresponds to the degrees of freedom $\nu$. Student-U and Student-L parameters estimates use respectively $\hat{\lambda}_{U}$ and $\hat{\lambda}_{L}$.

\begin{tabular}{|c|c|c|c|c|c|}
\hline \multicolumn{2}{|r|}{ Copula } & \multicolumn{3}{|c|}{ Parameters } & \multirow{2}{*}{$\frac{D_{2}}{55.82}$} \\
\hline \multirow{5}{*}{$\left(\left(Z_{1}\right)_{t},\left(Z_{2}\right)_{t}\right)$} & Gum + Surv Gum & 2.669 & 1.813 & 0.429 & \\
\hline & Gum + Clay & 5.506 & 0.913 & 0.348 & 32.81 \\
\hline & Surv Clay + Surv Gum & 0.901 & 5.919 & 0.651 & 36.55 \\
\hline & Surv Clay + Clay & 0.961 & 3.182 & 0.620 & 23.45 \\
\hline & Student & 0.442 & 2.665 & - & 9.58 \\
\hline \multirow{6}{*}{$\left(\left(Z_{1}\right)_{t},\left(Z_{3}\right)_{t}\right)$} & Gum + Surv Gum & 2.202 & 1.349 & 0.296 & 9.68 \\
\hline & Gum + Clay & 1.361 & 1.067 & 0.556 & 4.09 \\
\hline & Surv Clay + Surv Gum & 0.921 & 1.442 & 0.396 & 5.74 \\
\hline & Surv Clay + Clay & 0.748 & 0.839 & 0.472 & 2.73 \\
\hline & Student-U & 0.374 & 4.104 & - & 16.14 \\
\hline & Student-L & 0.374 & 3.018 & - & 16.06 \\
\hline \multirow{6}{*}{$\left(\left(Z_{2}\right)_{t},\left(Z_{3}\right)_{t}\right)$} & Gum + Surv Gum & 1.800 & 1.713 & 0.443 & 34.98 \\
\hline & Gum + Clay & 1.391 & 1.115 & 0.471 & 6.57 \\
\hline & Surv Clay + Surv Gum & 0.786 & 1.644 & 0.402 & 11.72 \\
\hline & Surv Clay + Clay & 0.734 & 0.992 & 0.428 & 4.38 \\
\hline & Student-U & 0.384 & 4.648 & - & 15.91 \\
\hline & Student-L & 0.384 & 2.564 & - & 19.50 \\
\hline
\end{tabular}

\subsection{The AIC diagnostic}

In this part we estimate the copulas' parameters maximizing the pseudo log-likelihood function. It is given for the generic parameter $\psi$ introduce in Section 4.1, by:

$$
\tilde{\psi}=\arg \max _{\psi} \sum_{k=1}^{N} \log L\left(\psi ; \hat{F}_{i N}\left(z_{i k}\right), \hat{F}_{j N}\left(z_{j k}\right)\right),
$$

with $L(\psi ; u, v)=\frac{\partial^{2}}{\partial u \partial v} C(u, v)$. This estimator $\tilde{\psi}$ is consistent and asymptotically Gaussian, see Genest et al. (1995) and Shih and Louis (1995) for details. This maximisation provides the Akaike Information Criteria (AIC), see Akaike (1974). We use it to discriminate between the different copulas: the adjustment obtained with the mimimum value of the AIC yields the best copula. This approach is not new and was already used by Breymann et al. (2003) on intraday FX rate data. It provides consistent estimators in an independent setting. We know that we are not in this context. Nevertheless, if we employ any filter on the data sets, it is very difficult to obtain a sequence of independent residual on which we can seriously work (details are available in Caillault and Guégan (2004)). Thus we decided to work directly on the log-returns and we used the results in a comparative way with the $D_{2}$ diagnostic.

Table 7 gives the maximum likelihood estimates obtained for the copulas' parameters and their standard errors computed with the Jackknife methodology. It also provide the AIC criterion calculated for the different copulas adjusted on the markets pairs. With 
this method, we retain, as the best copula, a Student copula for the pairs $\left(\left(Z_{1}\right)_{t},\left(Z_{2}\right)_{t}\right)$ and $\left(\left(Z_{2}\right)_{t},\left(Z_{3}\right)_{t}\right)$ and a convex linear combination of Gumbel with a survival Gumbel copula for the pair $\left(\left(Z_{1}\right)_{t},\left(Z_{3}\right)_{t}\right)$. We observe that for the pair $\left(\left(Z_{1}\right)_{t},\left(Z_{2}\right)_{t}\right)$ we obtain the same class of copulas as with $D_{2}$ distances, but for the pairs $\left(\left(Z_{1}\right)_{t},\left(Z_{3}\right)_{t}\right)$ and $\left(\left(Z_{2}\right)_{t},\left(Z_{3}\right)_{t}\right)$ the adjustment is not the same. We report in Table 8 the estimates of the upper and lower tail dependence parameters for these copulas, computed with expressions (2.2), (2.5) and (2.6).

Table 7. Parameter estimates, standard errors between brackets and Akaike's criterion values for each copula and each pair of markets. Standard errors are computed using the Jackknife methodology (100 observations of 4033 are omitted at each computation). For the convex linear combinations of Archimedean copulas the three parameters are $\theta_{1}, \theta_{2}, \omega$. For the Student copula the first parameter is the Pearson's correlation and the second one corresponds to the degrees of freedom.

\begin{tabular}{llrr}
\hline & Copula & Parameters & AIC \\
\hline Student & $0.444(0.014): 3.966(0.277)$ & -1079.68 \\
Frank & $2.926(0.124)$ & -804.72 \\
Gum + Surv Gum & $1.132(0.043): 1.873(0.084): 0.464(0.052)$ & -1056.97 \\
Gum + Clay & $1.284(0.131): 1.408(0.075): 0.600(0.029)$ & -1030.15 \\
$\left(\left(Z_{1}\right)_{t},\left(Z_{2}\right)_{t}\right)$ & Gum + Surv Joe & $1.917(0.809): 1.392(0.860): 0.383(0.269)$ & -1045.65 \\
& Joe + Clay & $1.409(0.072): 1.203(0.163): 0.469(0.043)$ & -1025.10 \\
& Joe + Surv Joe & $1.573(0.193): 1.831(0.189): 0.428(0.075)$ & -1027.40 \\
Surv Clay + Clay & $0.562(0.159): 1.104(0.265): 0.464(0.071)$ & -1004.97 \\
Surv Clay + Surv Gum & $2.058(0.703): 1.361(0.022): 0.148(0.039)$ & -1030.76 \\
\hline Student & $0.373(0.014): 4.381(0.317)$ & -708.62 \\
Frank & $2.406(0.107)$ & -557.99 \\
Gum + Surv Gum & $2.472(0.205): 1.143(0.026): 0.274(0.041)$ & -715.21 \\
Gum + Clay & $1.189(0.163): 1.662(0.100): 0.704(0.014)$ & -659.57 \\
$\left(\left(Z_{1}\right)_{t},\left(Z_{3}\right)_{t}\right)$ & Gum + Surv Joe & $2.223(0.107): 1.187(0.031): 0.338(0.027)$ & -714.39 \\
& Joe + Clay & $1.256(0.060): 1.178(0.293): 0.555(0.621)$ & -647.75 \\
Joe + Surv Joe & $2.811(0.387): 1.313(0.032): 0.259(0.029)$ & -676.29 \\
Surv Clay + Clay & $2.181(0.437): 0.367(0.036): 0.242(0.034)$ & -663.79 \\
Surv Clay + Surv Gum & $2.770(0.666): 1.217(0.022): 0.180(0.033)$ & -684.07 \\
\hline Student & $0.387(0.015): 3.482(0.280)$ & -836.68 \\
Frank & $2.488(0.134)$ & -586.06 \\
Gum + Surv Gum & $1.019(0.036): 2.105(0.150): 0.522(0.058)$ & -820.59 \\
Gum + Clay & $1.152(0.081): 1.972(0.035): 0.652(0.017)$ & -768.75 \\
& Gum + Surv Joe & $2.449(0.174): 1.233(0.194): 0.309(0.046)$ & -821.94 \\
& Joe + Clay & $1.183(0.031): 1.633(0.243): 0.563(0.041)$ & -760.54 \\
Joe + Surv Joe & $2.843(0.340): 1.372(0.200): 0.237(0.065)$ & -769.94 \\
Surv Clay + Clay & $2.275(0.371): 0.432(0.034): 0.221(0.025)$ & -737.45 \\
Surv Clay + Surv Gum & $3.005(0.299): 1.256(0.078): 0.151(0.032)$ & -771.35 \\
\hline
\end{tabular}

\subsection{Comparison of the two methods}

In this subsection we compare the results obtained using the two different approaches to find the bivariate distribution which characterizes each markets pair. 
Table 8. Tail dependent parameters for each pair of markets adjusted using the AIC criteria.

\begin{tabular}{rrrr}
\hline & Model & $\tilde{\lambda}_{U}$ & $\tilde{\lambda}_{L}$ \\
\hline$\left(\left(Z_{1}\right)_{t},\left(Z_{2}\right)_{t}\right)$ & Student & 0.225 & 0.225 \\
$\left(\left(Z_{1}\right)_{t},\left(Z_{3}\right)_{t}\right)$ & Gum + Surv Gum & 0.182 & 0.119 \\
$\left(\left(Z_{2}\right)_{t},\left(Z_{3}\right)_{t}\right)$ & Student & 0.224 & 0.224 \\
\hline
\end{tabular}

(i) For the pair $\left(\left(Z_{1}\right)_{t},\left(Z_{2}\right)_{t}\right)$, using the two approaches, a Student copula can be retained as the joint distribution. Nevertheless the estimates obtained for the parameter $\nu$ are not the same. Using the empirical approach, we obtained $\hat{\nu}=2.665$ and with the maximum likelihood approach $\tilde{\nu}=3.966$. We observe also that $\hat{\lambda}_{U}=0.302$ and $\hat{\lambda}_{L}=0.305$ using the "Naïve" estimator and $\tilde{\lambda}_{U}=\tilde{\lambda}_{L}=0.225$ when we derived these values from the maximum likelihood estimator. Thus, the first approach provides higher values for the tail dependent parameters. This difference can be important if we want to use these adjustments in order to propose a risk's measure.

(ii) For the pair $\left(\left(Z_{1}\right)_{t},\left(Z_{3}\right)_{t}\right)$ the analysis is more complicated because the two approaches suggest two different copulas. With the first approach we retain a convex linear combination of survival Clayton + Clayton copulas whereas with the second one we select a convex linear combination of survival Gumbel + Gumbel copulas. Tail dependence parameter's estimate using the "Naïve" approach provide the value $\hat{\lambda}_{U}=0.187$ and $\hat{\lambda}_{L}=0.231$ whereas the maximum likelihood provides $\tilde{\lambda}_{U}=0.182$ and $\tilde{\lambda}_{L}=0.119$. Contrary to the second approach the first method gives more weight on the lower tail.

(iii) For the pair $\left(\left(Z_{2}\right)_{t},\left(Z_{3}\right)_{t}\right)$ we conclude again to different copulas with the two approaches. With the "Naïve" method we retain the convex linear combination of survival Clayton with a Clayton copula whereas we conclude to Student copula with the maximum likelihood estimator. The "Naïve" approach gives higher value for the lower tail dependence than for the upper tail dependence: $\hat{\lambda}_{U}=0.167$ and $\hat{\lambda}_{L}=0.231$. With the maximum likelihood estimator we obtain $\tilde{\lambda}_{U}=\tilde{\lambda}_{L}=0.224$.

It is important to note that the two approaches contain is their formulations limitations that we discuss now:

- The First Approach. The estimates of $\hat{\lambda}_{U}$ and $\hat{\lambda}_{L}$ are based on the choice of the parameter $i_{0}$. This approximation is also used to estimate $\psi$ and in fine to choose the copula. This is the main limitation of this approach. On the other hand, this approach is really interesting because we do not choose a copula a priori and we do not make any independent assumption on the data. Indeed we derive the copula, in fine, using the tail behavior of the data that we want to investigate.

- The Second Approach. After an a priori choice of copulas, we estimate the parameters $\psi$ maximizing the pseudo log-likelihood function defined by (4.3). Maximum of this function is not always easy to obtain. In order to obtain the 
convergence of the algorithms we need to initialize it graphically. Thus, here we find the classical problem with the maximisation of the likelihood function knowing that we need to choose a copula a priori. Moreover, we also assume that the observations are independent which is a strong assumption.

Thus, the debate concerning the choice of the method to estimate a copula characterizing pairs of data is opened.

In a first analysis, we can nevertheless use these approaches to understand co-movements between Asian markets. If we keep in mind the results obtained using the first approach, we observe that the bivariate distribution which characterizes the Thai and Malaysian markets can be explained with a Student copula. This means that the behavior of these two markets is the same and they probably move in the same direction with respect to extreme shocks (positive and negative shocks). The markets pairs, Thai and Indonesian, and, Malaysian and Indonesian are explained with a convex linear combination of survival Clayton with a Clayton copula. For these two markets pairs the lower tail dependence parameter is bigger than the upper tail dependence one. It means that we have co-movement for these two markets pairs when occur very high negative shocks.

\section{Measure of risk for an Asian portfolio}

In this section we compute the Value at Risk (VaR) for a portfolio composed by two Asian indexes. We take into consideration the notion of copulas and the two empirical approaches of the previous section.

We define the value of the portfolio $\mathcal{P}$ at time $t+1$ as:

$$
P(t+1)=P(t) \exp \left(a_{i} R_{i}(t+1)+a_{j} R_{j}(t+1)\right),
$$

where $R_{i}$ and $R_{j}$ are the log-returns of the assets $i$ and $j$ with $i, j=1,2,3$ and $j>i$. $a_{i}$ and $a_{j}$ are respectively the weight associated to $R_{i}$ and $R_{j}$. We recall that these logreturns correspond to the Asian indexes whose marginals are denoted $F_{i}\left(. ; \alpha_{i}\right), i=1,2,3$ ( $\alpha_{i}$ are the parameters of the probability distribution function $F_{i}$ ). Thus, the portfolio's log-returns at time $t+1$ is given by:

$$
R_{p}(t+1)=a_{1} R_{1}(t+1)+a_{2} R_{2}(t+1) .
$$

To build a risk measure for the portfolio $\mathcal{P}$, we need to compute $R_{p}$. Thus we are going to construct its associated hyperplane $\mathcal{H}$ in $[0,1]^{2}$. Let $\left\{\left(u, v\left(\alpha, R_{p}, u\right)\right) \mid u \in[0,1]\right\}$ a point in $\mathcal{H}$ with $\alpha$ the set of the marginal's parameters (for instance $\alpha=\left(\alpha_{1}, \alpha_{2}\right)$ ). In our case, the marginals are Laplace distribution functions whose parameters are estimated in Section 4. Thus, this hyperplane depends on variables $(u, v)$. If we fix $u \in[0,1]$, then $v$ is analytically computed by the following relationship:

$$
v=F_{2}\left(\alpha_{2} ; \frac{R_{p}}{a_{2}}-\frac{a_{1}}{a_{2}} F_{1}^{-1}\left(\alpha_{1} ; u\right)\right) .
$$


The associated VaR of $R_{p}$ corresponds to the percentile of the $R_{p}$ 's distribution function. This percentile is measured by the copula's volume on plane:

$$
H_{2}\left(\alpha, R_{p}\right):=\left\{(u, v) \in[0,1] \times\left[0, v^{*}\left(\alpha, R_{p}, u\right)\right]\right\} .
$$

This VaR is computed numerically by solving the following integral which is the profit and lost $G_{P \& L}$ distribution function:

$$
G_{P \& L}\left(R_{p}\right)=\int_{H_{2}\left(\alpha, R_{p}\right)} c(u, v) d u d v,
$$

where $c$ is the probability density function of a copula $C$. For a given level $\beta=G_{P \& L}\left(R_{p}\right)$ we must solve equation (5.3). But this latter expression is not always easy to compute. So, we are going to use a Monte Carlo method to obtain this VaR. Usually in Finance, market actors are interested in computing this risk's measure for $\beta=1 \%, 5 \%, 10 \%$ and $\beta=90 \%, 95 \%, 99 \%$. We propose three methods to compute the VaR for a portfolio composed by two Asian indexes with $a_{1}=a_{2}=1 / 2$. The methods we considered are the historical simulation and the Monte Carlo VaR using the copulas adjusted with the two approaches developed in Section 4. We denote MC VaR 1, the VaR computed using the copulas of the "Naïve" approach and MC VaR 2 the VaR computed using the copulas selected with the AIC criteria (see Caillault and Guégan (2004) for more details).

The results for the VaR in Table 9 are provided with their standard error in brackets. To obtain the latter we used a bootstrap method with the historical VaR. Standard error of MC VaR are computed with 1000 VaR calculated from simulated sample of length 2000. The obtained results are close whatever method used and some of them are not statistically different. Nevertheless, when we consider the results more precisely, we observe that the portfolio's losses and profits can be more significant in certain cases. For instance, the values obtained using the Historical method for the portfolio $\left(\left(Z_{1}\right)_{t},\left(Z_{2}\right)_{t}\right)$ are larger (for $\beta=99 \%$ ) and lower (for $\beta=1 \%$ ) than with the MC VaR methods.

We note also that the VaR obtained with MC-VaR1 for portfolios $\left(\left(Z_{1}\right)_{t},\left(Z_{3}\right)_{t}\right)$ and $\left(\left(Z_{2}\right)_{t},\left(Z_{3}\right)_{t}\right)$ are respectively lower and upper than those computed with the $\mathrm{EH}$ and MC-VaR2 approaches.

\section{Conclusion}

In this paper we propose two methods which allow us to estimate the copula characterizing the bivariate distribution function of a pair of markets. One method privileges the extreme behavior of the bivariate distribution function of the pair of markets. The second one is more classic and is based on the estimates of the copulas' parameter using a pseudo log-likelihood method. Then, we used the two different approaches to propose risk's measures for a portfolio composed with the different pair of markets. To give specific value for the Value at Risk necessitates a degree of certainty looking at the 
Table 9. VaR computations using historical data and Monte Carlo simulations with copulas estimated with the two approaches introduced in Section 4.

\begin{tabular}{|r|r|r|r|r|}
\hline & Risk's level & Historical VaR & MC VaR 1 & MC VaR 2 \\
\hline \multirow{5}{*}{$\left(\left(Z_{1}\right)_{t},\left(Z_{2}\right)_{t}\right)$} & $1 \%$ & $-5.03 \%(0.26 \%)$ & $-4.78 \%(0.29 \%)$ & $-4.70 \%(0.27 \%)$ \\
& $5 \%$ & $-2.55 \%(0.09 \%)$ & $-2.74 \%(0.11 \%)$ & $-2.76 \%(0.11 \%)$ \\
& $10 \%$ & $-1.74 \%(0.06 \%)$ & $-1.91 \%(0.07 \%)$ & $-1.95 \%(0.07 \%)$ \\
& $90 \%$ & $1.62 \%(0.03 \%)$ & $1.89 \%(0.07 \%)$ & $1.93 \%(0.07 \%)$ \\
& $95 \%$ & $2.23 \%(0.07 \%)$ & $2.71 \%(0.11 \%)$ & $2.74 \%(0.11 \%)$ \\
& $99 \%$ & $4.90 \%(0.24 \%)$ & $4.77 \%(0.29 \%)$ & $4.71 \%(0.28 \%)$ \\
\hline \multirow{5}{*}{$\left(\left(Z_{1}\right)_{t},\left(Z_{3}\right)_{t}\right)$} & $1 \%$ & $-5.30 \%(0.24 \%)$ & $-5.43 \%(0.16 \%)$ & $-5.18 \%(0.24 \%)$ \\
& $5 \%$ & $-2.76 \%(0.09 \%)$ & $-3.26 \%(0.08 \%)$ & $-3.12 \%(0.10 \%)$ \\
& $10 \%$ & $-2.84 \%(0.05 \%)$ & $-2.34 \%(0.06 \%)$ & $-2.31 \%(0.07 \%)$ \\
& $90 \%$ & $1.70 \%(0.05 \%)$ & $2.31 \%(0.06 \%)$ & $2.24 \%(0.07 \%)$ \\
& $95 \%$ & $2.39 \%(0.08 \%)$ & $3.20 \%(0.09 \%)$ & $3.20 \%(0.06 \%)$ \\
$\left(\left(Z_{2}\right)_{t},\left(Z_{3}\right)_{t}\right)$ & $99 \%$ & $5.43 \%(0.26 \%)$ & $5.30 \%(0.25 \%)$ & $5.38 \%(0.23 \%)$ \\
\hline & $1 \%$ & $-5.45 \%(0.34 \%)$ & $-5.48 \%(0.21 \%)$ & $-5.32 \%(0.25 \%)$ \\
& $5 \%$ & $-2.24 \%(0.10 \%)$ & $-3.24 \%(0.08 \%)$ & $-3.09 \%(0.09 \%)$ \\
& $10 \%$ & $-1.63 \%(0.05 \%)$ & $-2.31 \%(0.06 \%)$ & $-2.18 \%(0.06 \%)$ \\
& $90 \%$ & $1.52 \%(0.04 \%)$ & $2.28 \%(0.05 \%)$ & $2.16 \%(0.06 \%)$ \\
& $95 \%$ & $2.23 \%(0.08 \%)$ & $3.15 \%(0.07 \%)$ & $3.07 \%(0.09 \%)$ \\
& $99 \%$ & $5.19 \%(0.36 \%)$ & $5.18 \%(0.16 \%)$ & $5.29 \%(0.25 \%)$ \\
\hline
\end{tabular}

distribution of the portfolio. But the comparison between the two approaches considered here do not provide a conclusion. For us, the choice of the best method to find a copula characterizing a vector is always an open-ended problem. We also propose new estimators for the tail behavior of a tail of markets and we provide in Table 1, informative results on the properties of the copulas with respect to their tail behavior. The knowledge of these properties is fondamental from an empirical point of view. The theoretical properties of the "Naïve" estimators that we introduce in Section 3 need to be developed and will be the subject of another paper. The ideas developed in this paper have to be extended in a higher setting. Finally, questions regarding copula parameters dynamic need to be asked. This issue has been already developed by Patton (2001) in the Gaussian and Joe-Clayton case. These topics must be explored and will be the purpose of future research.

[1] H. Akaike. A new look to the statistical model identification. IEEE Transactions on Automatic Control, AC-19:716-723, (1974).

[2] Berntsen, J., T. O. Espelid, and A. Genz. An adaptive algorithm for the approximate calculation of multiple integrals. ACM Transactions on Mathematical Software, 17:437-451, (1991a).

[3] Berntsen, J., T. O. Espelid, and A. Genz. An adaptive multidimensional integration routine for a vector of integrals. ACM Transactions on Mathematical Software, 17:452-456, (1991b).

[4] Boyer, B.H., M.S. Gibson, and M. Loretan. Pitfalls in tests for changes in correlations. Federal Reserve Board, IFS Discussion Paper No597R, (1999).

[5] Breymann, W., A. Dias, and P. Embrechts. Dependence structures for multivariate high-frequency data in finance. Quantitative Finance, 3(1):1-14, (2003).

[6] Caillault, C., and D. Guégan. Empirical estimation of tail dependence using copulas. application to asian markets. Working Paper No 05-2003, IDHE-MORA, Ecole Normale Supérieure de 
Cachan, France, (2003).

[7] Caillault, C., and D. Guégan. Forecasting var and expected shortfall using dynamical systems: A risk management stategy. Working Paper No 07-2004, IDHE-MORA, Ecole Normale Supérieure de Cachan, France, (2004).

[8] Cambanis, S., S. Huang, and G. Simons. On the theory of elliptical contoured distributions. Journal of Multivariate Analysis, 11:368-385, (1981).

[9] Coles, S., J. Heffernan, and J. Tawn. Dependence measures for extreme value analysis. Extremes, 2(4):339-365, (1999).

[10] P. Deheuvels. La fonction de dépendance empirique et ses propriétés - un test non paramétrique d'indépendance. Académie Royale de Belgique - Bulletin de la classe des sciences - 5o Série, 65:274-292, (1979).

[11] Efron, B., and R. J. Tibshirani. An Introduction to the Bootstrap. Chapman and Hall, New York, (1993).

[12] Embrechts, P., A. McNeil, and D. Straumann. Correlation: Pitfalls and alternatives, a short, non-technical article. RISK Magazine, pages 69-71, May (1999).

[13] Embrechts, P., A. McNeil, and D. Strausmann. Correlation and dependence in risk management: properties and pitfalls. In: Risk Management: Value at Risk and Beyond, ed. M.A.H. Dempster, Cambridge University Press, Cambridge, pages 176-223, (2002).

[14] Fang, H.-B., K.-T. Fang, and S. Kotz. The meta-elliptical distributions with given marginals. Journal of Multivariate Analysis, 82:1-16, (2002).

[15] Frees, E. W., and E. A. Valdez. Understanding relationships using copulas. North American Actuarial Journal, 2:1-25, (1998).

[16] Genest, C., K. Ghoudi, and L.-P Rivest. A semiparametric estimation procedure for dependence parameters in multivariate families of distributions. Biometrica, 82(3):543-552, (1995).

[17] Genest, C., and R.-J. MacKay. Copules archimédiennes et familles de lois bidimentionnelles dont les marges sont données. Canadian Journal of Statistics, 14:145-159, (1986a).

[18] Genest, C., and R.-J. MacKay. The joy of copulas: Bivariate distribution with uniforms marginals. American Statistician, 40:280-283, (1986b).

[19] Georges, P., A-G. Lamy, E. Nicolas, G. Quibel, and T. Roncalli. Multivariate survival modelling: a unified approach with copulas. Working Paper, Groupe de Recherche Opérationnelle du Crédit Lyonnais, (2001).

[20] J. Heffernan. A directory of coefficients of tail dependence. Extremes, 3:279-290, (2000).

[21] H. Joe. Multivariate Models and Dependence Concepts. Chapman \& Hall, London, (1997).

[22] Ledford, T.-P., and J.-A. Tawn. Statistics for near independence in multivariates extreme values. Biometrika, 83:169-187, (1996).

[23] Ledford, T.-P., and J.-A. Tawn. Concomitant tail behavior for extremes. Advances in Applied Probability, 30:197-215, (1998).

[24] Lindskog, F., A. McNeil, and H. Schmoch. A note on kendall's tau for elliptical distributions. ETH preprint, (2001).

[25] Malevergne, Y., and D. Sornette. How to account for extreme comovements between stocks and the markets? Working paper, (2001).

[26] Malevergne, Y., and D. Sornette. Testing the gaussian copula hypothesis for financial assets dependences. Quantitative Finance, 4(3):231-250, (2003).

[27] D. Oakes. Multivariate survival distribution. Journal of Nonparametric Statistics, 3:343-354, (1994).

[28] A. J. Patton. Modelling time-varying exchange rate dependence using the conditionnal copula. Working Paper, University of California, San Diego, (2001).

[29] Shih, J.H., and T.A. Louis. Inferences on the association parameter in copula models for bivariate survival data. Biometrics, 51:1384-1399, (1995).

[30] A. Sklar. Fonctions de répartition à n dimensions et leurs marges. Publications de l'Institut de Statistique de L'Université de Paris, 8:229-231, (1959). 